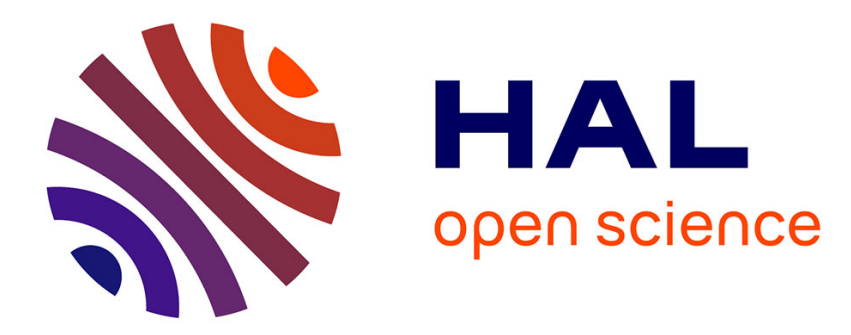

\title{
Temperature Dependent Model for Hole Effective Mass in Heavily Doped p-type SiGe
}

S. Sokolic, S. Amon

\section{To cite this version:}

S. Sokolic, S. Amon. Temperature Dependent Model for Hole Effective Mass in Heavily Doped p-type SiGe. Journal de Physique IV Proceedings, 1996, 06 (C3), pp.C3-137-C3-142. 10.1051/jp4:1996321 . jpa-00254239

\section{HAL Id: jpa-00254239 https://hal.science/jpa-00254239}

Submitted on 1 Jan 1996

HAL is a multi-disciplinary open access archive for the deposit and dissemination of scientific research documents, whether they are published or not. The documents may come from teaching and research institutions in France or abroad, or from public or private research centers.
L'archive ouverte pluridisciplinaire HAL, est destinée au dépôt et à la diffusion de documents scientifiques de niveau recherche, publiés ou non, émanant des établissements d'enseignement et de recherche français ou étrangers, des laboratoires publics ou privés. 


\title{
Temperature Dependent Model for Hole Effective Mass in Heavily Doped p-type SiGe
}

\author{
S. Sokolić and S. Amon
}

Faculty of Electrical Engineering, University of Ljubljana, Tržaška 25, 1000 Ljubljana, Slovenia

\begin{abstract}
As a consequence of compressive strain and alloying, the hole effective mass in $p$-type strained $\mathrm{SiGe}$ differs significantly from its $\mathrm{Si}$ value. Influencing the carrier concentrations in the base, the hole effective mass affects the base transport properties of npn SiGe HBTs. A new model for hole effective mass is presented in this work, which takes into consideration the dependence on temperature, doping concentration and germanium fraction. The model is based on experimental data and theoretical studies of the valence band structure supported by the detailed numerical analysis of hole effective mass. It is suitable for efficient analysis and optimization of SiGe HBTs, and can be tuned to the measurements of carrier transport. The model is valid in the temperature range from $77 \mathrm{~K}$ to $300 \mathrm{~K}$, for doping concentrations up to $10^{20} \mathrm{~cm}^{-3}$ and for germanium fractions up to 0.2 .
\end{abstract}

\section{INTRODUCTION}

Extensive work in the field of design, fabrication and modelling of npn SiGe HBTs has been performed in the last few years [1]. As expected, the transport characteristics of npn SiGe HBTs depend significantly on the properties of base $p$-type strained SiGe layers [1]. Since SiGe HBTs are suitable for low temperature operation [2], appropriate models describing properties of these devices down to $77 \mathrm{~K}$ are necessary.

Effective density of states in the valence band of $\mathrm{SiGe}\left(N_{V, S i G e}\right)$ is lower than the effective density of states in the valence band of $\mathrm{Si}$ due to lower hole effective mass in SiGe [4]. $N_{V, S i G e}$ influences $p n$ product in the base and therefore affects the transport of carriers through the base $[1,2]$. The results of numerical simulation of SiGe HBTs demonstrate the importance of $N_{V, S i G e}$ model for accurate modelling of SiGe HBTs at low temperatures [3]. Since $N_{V, S i G e}$ is determined with hole effective mass $\left(m_{p}{ }^{*}\right)$ in $p$-type $\mathrm{SiGe}$, an appropriate model for $m_{p}{ }^{*}$ is inevitable for accurate modelling and optimization of SiGe HBTs. The purpose of this work is to present a new model for $m_{p}{ }^{*}$, which is applicable down to $77 K$ and takes into consideration the dependence on doping and $\mathrm{Ge}$ fraction.

\section{DEFINITION OF HOLE EFFECTIVE MASS}

Compared to silicon, the structure of the valence band in strained SiGe is modified due to compressive strain and alloying $[4,5]$. The heavy hole and the light hole bands split, where the heavy hole band moves up and the light hole band moves down in the energy diagram. Both bands, together with the split-off band are highly distorted and non-parabolic. The carrier concentration effective mass can be defined to take into account the influence of non-parabolic valence band [4]. The correct relationship between Fermi 
level and the majority carrier concentration can thus be accomplished without loss of validity of Fermi integral. For this case the hole effective mass $\left(m_{p}{ }^{*}\right)$ is defined with (1) [4]:

$$
m_{p}^{*}\left(E_{F p}, T, x_{G e}\right)^{3 / 2}=\frac{(k T)^{-3 / 2}}{\mathrm{~F}_{1 / 2}\left(\frac{E_{V}-E_{F p}}{k T}\right)} \int_{0}^{\infty} \frac{m_{V, D O S}^{*}\left(E, x_{G e}\right)^{3 / 2} \sqrt{E}}{1+\exp \left(\frac{E-E_{V}+E_{F p}}{k T}\right)} d E,
$$

where $x_{G e}$ is Ge fraction and $m_{V, D O S}{ }^{*}$ is the density of states effective mass whose energy dependence results from the non-parabolic density of states in the valence band $\left(g_{y}\right)$ :

$$
g_{V}\left(E, x_{G e}\right)=\frac{\sqrt{2} m_{V, D O S}^{*}\left(E, x_{G e}\right)^{3 / 2} \sqrt{E_{V}-E}}{\pi^{2} \hbar^{3}} .
$$

According to its definition (1), $m_{p}{ }^{*}$ is a function of temperature $(T)$, Ge fraction and in degenerated material where Boltzmann statistics is not valid also a function of Fermi level (doping concentration).

If the density of states in strained $\mathrm{SiGe}$ is known, $m_{p}^{*}\left(T, N_{A}, x_{G e}\right)$ can be obtained by means of (1) and (2). Nevertheless, the evaluation of $m_{p}{ }^{*}$ by means of (1) appears to be inappropriate for device modelling due to its numerical complexity. Furthermore, in this case $g_{v}\left(E, x_{G e}\right)$ would be the independent model parameter and it would be difficult to tune the model to the measurements of carrier transport, what is an important disadvantage of this approach. Finally, the hole effective mass for lightly doped $p$ type Si calculated by means of $g_{v}\left(E, x_{G e}\right)$ determined by $\mathrm{Fu}$ et al. [5] - which seems to be the only $g_{V}\left(E, x_{G e}\right)$ model in the literature that can be efficiently used for the evaluation of $m_{p}{ }^{*}\left(T, N_{A}, x_{G e}\right)$ - is in disagreement with the model for hole effective mass suggested by Green as a result of comparison with experiments [6]. The modelling of SiGe based devices therefore requires a compact model for $m_{p}^{*}$ which is a function of temperature, doping concentration and Ge-fraction and whose dependence on these independent variables can be tuned to the experiments. Despite extensive work performed in relation to the modelling of hole effective mass in $\mathrm{SiGe}$, a model for $m_{p}{ }^{*}$ appropriate for device analysis can not be found in the present literature.

\section{NEW MODEL FOR HOLE EFFECTIVE MASS}

We propose a new model for the hole effective mass, which is defined with the following algorithm:

- As determined by $(1), m_{p}{ }^{*}$ in non-degenerate $\operatorname{SiGe}\left(m_{p}{ }^{*}\right)$, where Boltzmann statistics is valid, is not a function of doping and depends only on temperature and Ge fraction $\left(m_{p}{ }^{* 0}=m_{p}{ }^{* 0}\left(T, x_{G e}\right)\right)$. Hole effective mass in non-degenerate $p$-type Si $\left(m_{p}^{* 0}\left(T, x_{G e}=0\right)\right)$ was studied in [6]. Through the analysis of cyclotron resonance measurements at low temperatures and the measurements of intrinsic carrier concentration at high temperatures, Green [6] concluded that the model from [7] describes $m_{p}{ }^{* 0}(T)$ in silicon appropriately. Based on theoretical calculations of $m_{p}{ }^{* 0}\left(T, x_{G e}\right)$ from [4,5], we obtained the analytical expressions for $m_{p}{ }^{* 0}(T)$ also for different values of $x_{G e}$ (Fig.1). If $m_{p}{ }^{*}(T)$ is known, a temperature which represents a transition between degenerate and non-degenerate material $\left(T_{d e g}\right)$ can be found for every doping concentration and $\mathrm{Ge}$ fraction. The material is non-degenerate for $T>T_{\text {deg }}$, resulting in doping independence of hole effective mass $\left(T>T_{\text {deg }}: m_{p}{ }^{*}=m_{p}{ }^{*}\right) . T_{\text {deg }}$ is presented on Fig.2.

- $m_{p}{ }^{*}\left(N_{A}\right)$ should be known at one single low temperature. Low temperature photoluminescence measurements of Fermi level $[8,9,10,11]$ appear to be the most appropriate for the evaluation of $m_{p}{ }^{*}\left(N_{A}\right)$. In the case of $\mathrm{SiGe}$, where insufficient experimental data are available, the theory from [5] can be used. $m_{p}{ }^{*}\left(N_{A}\right)$ for silicon and SiGe is presented on Fig.3.

- We assume linear temperature dependence for $m_{p}{ }^{*}$ between low temperatures and $T_{d e g}$, where $m_{p}{ }^{*}$ cannot decrease with increasing $T$, and $m_{p}{ }^{* 0}$ represents the absolute lower boundary for $m_{p}{ }^{*}$. Detailed numerical modelling of $m_{p}{ }^{*}\left(T, N_{A}, x_{G e}\right)$ using $g_{V}\left(E, x_{G e}\right)$ model from [5] in (1) supports this assumption. - According to the theoretical calculations of $m_{p}{ }^{* 0}[4]$ and $m_{p}{ }^{*}[5]$ and the measurements of Fermi level 
[9], $m_{p}{ }^{*}$ decreases with increasing $x_{G e}$, which can be described with a double exponential decline. Consequently, to evaluate $m_{p}{ }^{*}$ for arbitrary $x_{G e}, m_{p}{ }^{*}$ should be known at $x_{G e}=0,0.1,0.2$ and 0.3 , and a system of four nonlinear equations should be solved to obtain $m_{p}{ }^{*}$ as a double exponential function of $x_{G e}$.

Fig. 4 shows a schematic representation of the proposed algorithm. Since closed-form analytical expressions for $m_{p}{ }^{*}$ can not be obtained for all conditions of interest, an efficient numerical routine based on proposed algorithm is built, allowing evaluation of $m_{p}^{*}$ for arbitrary $T, N_{A}$ and $x_{G e}$.

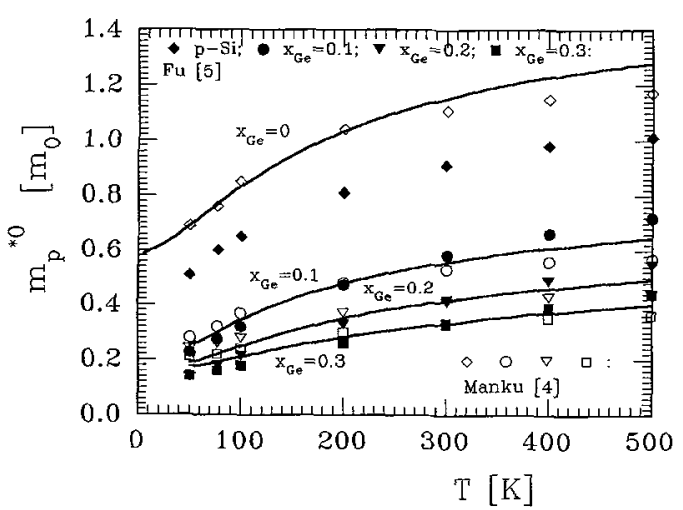

Figure 1: $m_{p}^{* 0}$ as a function of temperature for different $x_{G e}$.

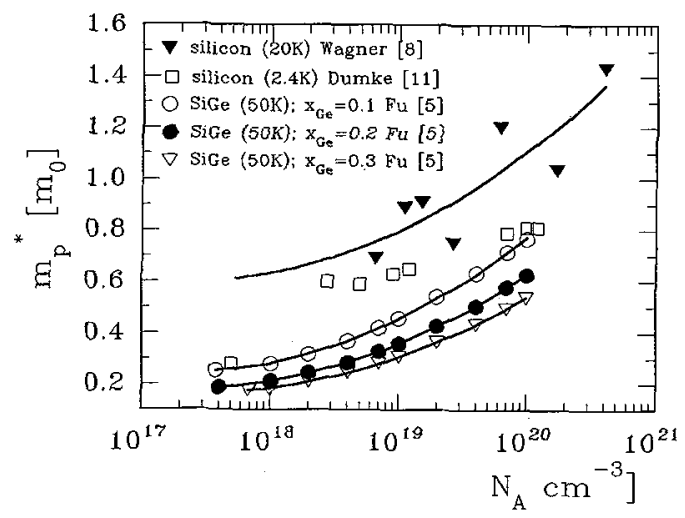

Figure 3: $m_{p}{ }^{*}$ as a function of doping concentration at low temperature for different $x_{G e}$. Wagner's data [8] were taken into account for $p$-type $\mathrm{Si}$.

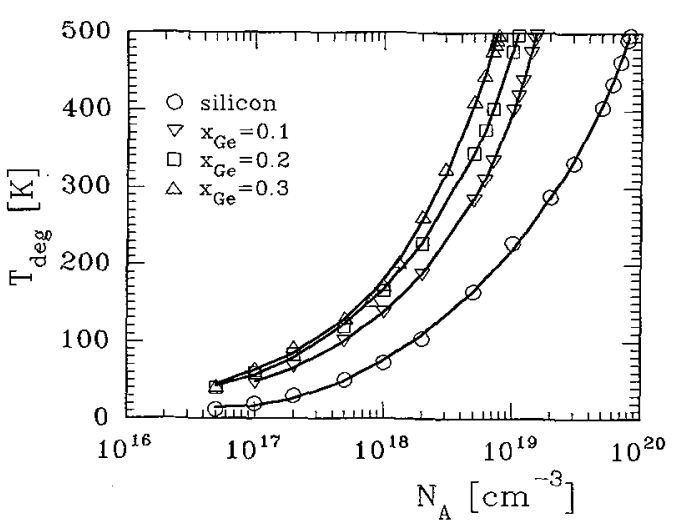

Figure 2: $T_{d e g}$ as a function of doping concentration for different $x_{G e}$.

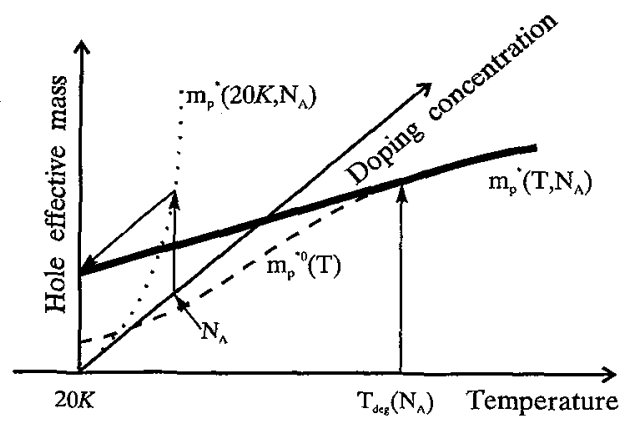

Figure 4: Schematic representation of proposed model. Dashed curve represents $m_{p}^{*}(T)$. Doted curve represents $m_{p}{ }^{*}\left(N_{A}\right)$ at $20 K$. Solid thick curve represents $m_{p}{ }^{*}(T)$ for one single value of $N_{A}$. 


\section{RESULTS AND DISCUSSION}

The hole effective mass as a function of temperature, doping and $\mathrm{Ge}$ fraction calculated with a new model is presented on Fig.5-7. As can be seen on Fig.5-7, the proposed model accounts for the main features of $m_{p}{ }^{*}$ : strong doping dependence at low temperatures, negligible temperature dependence at high doping concentrations and significant $\mathrm{Ge}$ dependence in the entire temperature and doping range of interest. Since $T_{\text {deg }}$ is higher than $77 \mathrm{~K}$ for the doping concentrations which are present in the base of SiGe HBTs, the doping dependence of $m_{p}{ }^{*}$ is important for the modelling of these devices at $77 \mathrm{~K}$.

Since $m_{p}^{*}\left(N_{A}\right)$ in $p$-type $\mathrm{Si}$ is obtained experimentally and employed $m_{p}^{* 0}\left(T, x_{G e}=0\right)$ model is also in agreement with the experiments (see discussion in [6]), we assume that for the case of $p$-type Si the proposed model is appropriate for $N_{A}<4 \cdot 10^{20} \mathrm{~cm}^{-3}$ and $4.2 \mathrm{~K}<T<500 \mathrm{~K}$. However, only Wagner's photoluminescence data [8] were used for the evaluation of $m_{p}{ }^{*}\left(N_{A}\right)$ (see Fig.3). Dumke's data [11] were neglected since they seem to be in disagreement with Wagner's data [8] which are obtained by more rigorous analysis of photoluminescence spectra, and are in disagreement with Green's results [6]. The situation is worse for the case of SiGe, where less experimental data are available. Nevertheless, we assume that the proposed model renders reasonable results in the following range of parameters: $77 K \leq T \leq 300 \mathrm{~K}, N_{A} \leq 10^{20} \mathrm{~cm}^{-3}$ and $x_{G e} \leq 0.2$. Since the proposed algorithm is suitable for tuning to the experimental data, the model can be further improved by additional measurements without loss of validity of the algorithm.

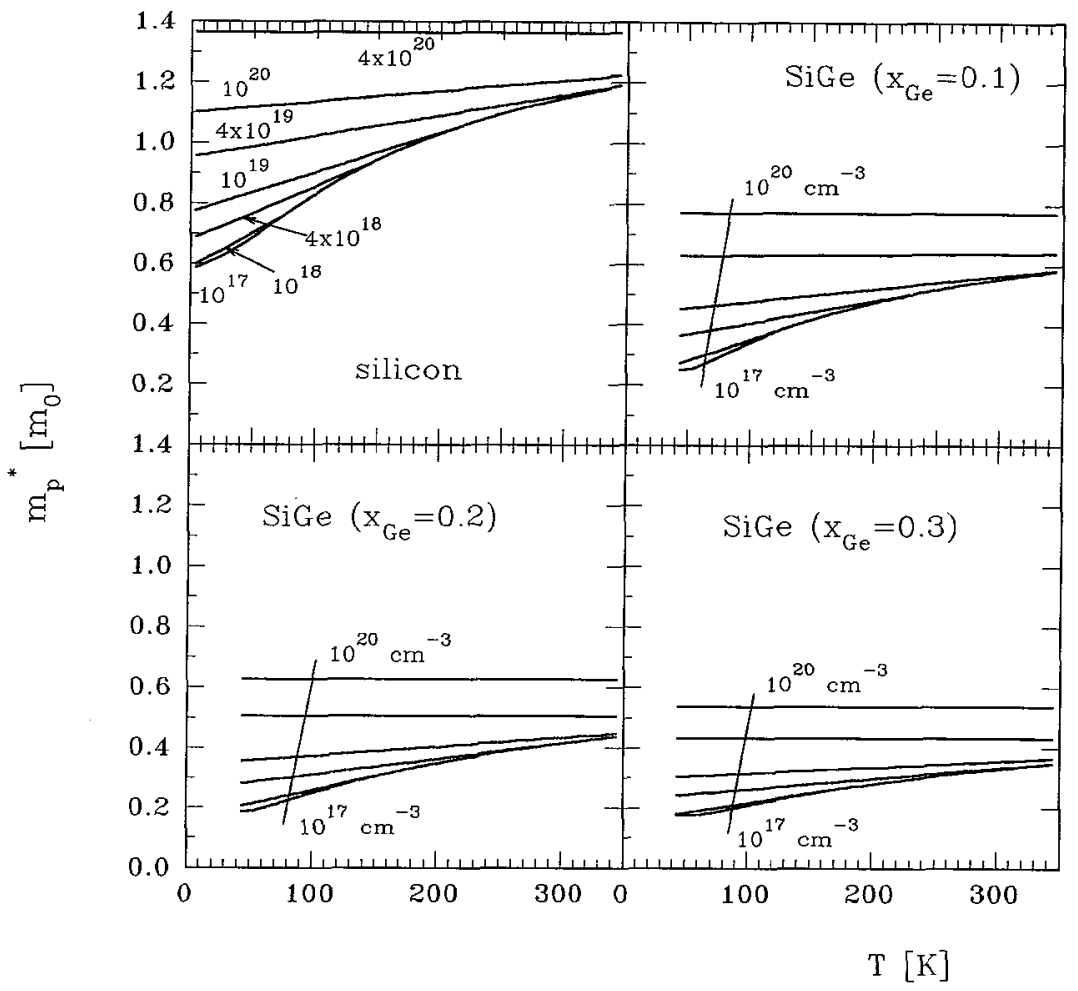

Figure 5: $m_{p}{ }^{*}$ as a function of temperature for different doping concentrations and $x_{G e}$ calculated with the proposed model. 


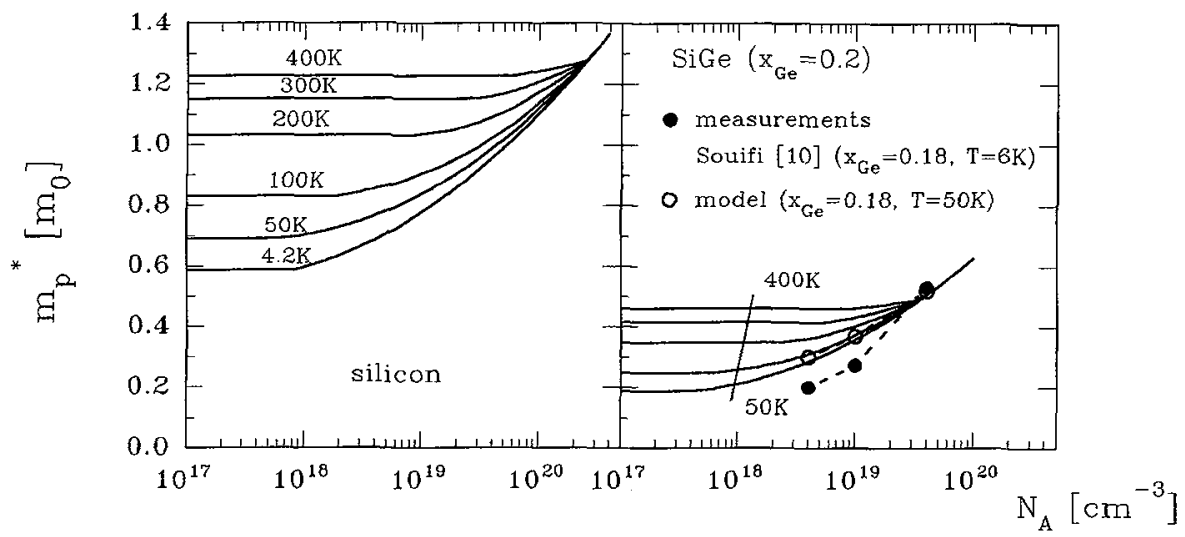

Figure 6: $m_{p}{ }^{*}$ as a function of doping concentration for different $T$ and $x_{G e}$ calculated with the proposed model. $m_{p}^{*}$ determined from photoluminescence measurements [10] $\left(x_{G e}=0.18, T=6 K, N_{A}=4 \cdot 10^{18} \mathrm{~cm}^{-3}, 10^{19} \mathrm{~cm}^{-3}\right.$ and $\left.4 \cdot 10^{19} \mathrm{~cm}^{-3}\right)$. represent model calculations for the same parameters except temperature $(T=50 K)$.

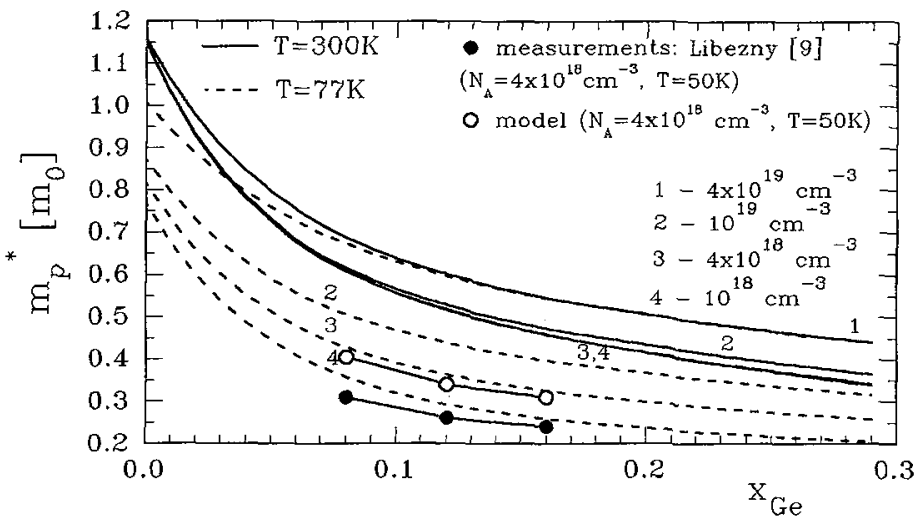

Figure 7: $m_{p}^{*}$ as a function of Ge-fraction for different $T$ and doping concentrations calculated with the proposed model. represents $m_{p}{ }^{*}$ determined from photoluminescence measurements [9] $\left(T=50 \mathrm{~K}, N_{A}=4 \cdot 10^{18} \mathrm{~cm}^{-3}, x_{G e}=0.08,0.12\right.$ and 0.16$)$. $O$ represent model calculations for the same parameters.

\section{CONCLUSIONS}

The model for hole effective mass in p-type strained SiGe, which takes into consideration the dependence on temperature, doping concentration and Ge fraction at acceptable level of complexity is presented for the first time. The proposed model is assumed to be valid in the temperature range from $77 \mathrm{~K}$ to $300 \mathrm{~K}$, and for doping concentrations and germanium fractions up to $10^{20} \mathrm{~cm}^{-3}$ and 0.2 , respectively. More experimental data for $\mathrm{SiGe}$ are necessary for the confirmation of assumed range of validity. Since the hole effective mass is important for the performance of SiGe HBTs, the proposed model could be useful for improved modelling of SiGe HBTs in the wide range of temperatures. 


\section{Acknowledgement}

This work was partially sponsored by the Ministry of Science and Technology of the Republic of Slovenia. The authors are grateful to $\mathrm{Y}$. Fu for providing the data for the density of states in the valence band of SiGe.

\section{References}

[1] Harame D. L., Comfort J. H., Cressler J. D., Crabbé E. F., Sun J. Y.-C., Meyerson B. S., Tice T., IEEE Trans. Electron Dev. 42 (1995) 455-468.

[2] Cressler J. D., "Status and trends in the cryogenic operation of SiGe bipolar technology", Symp. on Low Temp. Electron. and High Temp. Supercond., Reno, Nevada 21-26 May 1995, C. L. Claeys, S. I. Raider, R. K. Kirschman and W. D. Brown Eds. (ECS PV 95-9, The Electrochemical Society, Inc., Pennington) pp. 159-177.

[3] Richey D. M., Cressler J. D., Joseph A. J., Jaeger R. C., "Low-temperature modelling of SiGe HBTs using SCORPIO", Symp. on Low Temp. Electron. and High Temp. Supercond., Reno, Nevada 21-26 May 1995, C. L. Claeys, S. I. Raider, R. K. Kirschman and W. D. Brown Eds. (ECS PV 95-9, The Electrochemical Society, Inc., Pennington) pp. 178-188.

[4] Manku T., Nathan A., J. Appl. Phys. 69 (1991) 8414-8416.

[5] Fu Y., Jain S. C., Willander M., Loferski J. J., J. Appl. Phys. 74 (1993) 402-407; Fu Y., Grahn

K. J., Willander M., IEEE Trans. Electron Dev. 41 (1994) 26-31.

[6] Green M. A., J. Appl. Phys. 67 (1990) 2944-2954.

[7] Madarasz F. L., Lang J. E., Hemeger P. M., J. Appl. Phys. 52 (1981) 4646-4648; Lang J. E., Madarasz F. L., Hemeger P. M., J. Appl. Phys. 54 (1983) 3612.

[8] Wagner J., Solid-St. Electron. 28 (1985) 25-30.

[9] Libezny M., Jain S. C., Poortmans J., Caymax M., Nijs J., Mertens R., Werner K., Balk. P, Appl. Phys. Lett. 64 (1994) 1953-1955.

[10] Souifi A., Bremond G., Benyattou T., Guillot G., Appl. Phys. Lett. 62 (1993) 2986-2988.

[11] Dumke W. P., J. Appl. Phys. 54 (1983) 3200-3202. 